\title{
TITLE:
}

\section{Instantaneous vibrational frequencies of diffusing and desorbing adsorbates: CO/Pt(111).}

\section{$\operatorname{AUTHOR}(S)$ :}

Inoue, Ken-ichi; Watanabe, Kazuya; Matsumoto, Yoshiyasu

\section{CITATION:}

Inoue, Ken-ichi ...[et al]. Instantaneous vibrational frequencies of diffusing and desorbing adsorbates: CO/Pt(111).. The Journal of chemical physics 2012, 137(2): 024704.

ISSUE DATE:

2012-07

URL:

http://hdl.handle.net/2433/159717

RIGHT:

(C) 2012 American Institute of Physics 


\section{AIP $\begin{gathered}\text { molowanat } \\ \text { chemical Physics }\end{gathered}$}

\section{Instantaneous vibrational frequencies of diffusing and desorbing adsorbates: CO/Pt(111)}

Ken-ichi Inoue, Kazuya Watanabe, and Yoshiyasu Matsumoto

Citation: J. Chem. Phys. 137, 024704 (2012); doi: 10.1063/1.4733720

View online: http://dx.doi.org/10.1063/1.4733720

View Table of Contents: http://jcp.aip.org/resource/1/JCPSA6/v137/i2

Published by the American Institute of Physics.

\section{Additional information on J. Chem. Phys.}

Journal Homepage: http://jcp.aip.org/

Journal Information: http://jcp.aip.org/about/about_the_journal

Top downloads: http://jcp.aip.org/features/most_downloaded

Information for Authors: http://jcp.aip.org/authors

\section{ADVERTISEMENT}

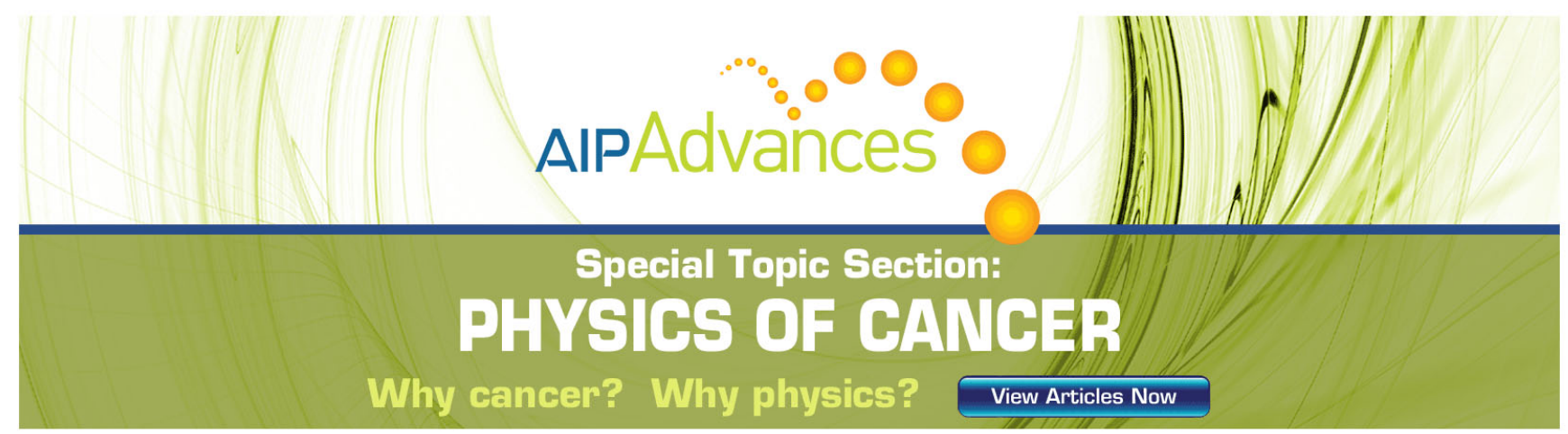




\title{
Instantaneous vibrational frequencies of diffusing and desorbing adsorbates: CO/Pt(111)
}

\author{
Ken-ichi Inoue, Kazuya Watanabe, and Yoshiyasu Matsumoto a) \\ Department of Chemistry, The Graduate School of Science, Kyoto University, Kyoto 606-8502, Japan
}

(Received 18 April 2012; accepted 21 June 2012; published online 13 July 2012)

\begin{abstract}
Electronic excitation of metal by intense laser pulses stimulates nuclear motions of adsorbates through nonadiabatic coupling, resulting in diffusion and desorption of adsorbates. These processes take place via precursor states: adsorbates whose vibrational modes with respect to substrate are highly excited. This paper reports the dynamics of precursor states of $\mathrm{CO}$ on $\mathrm{Pt}(111)$ probed by use of infrared-visible sum frequency generation with phase-sensitive detection, which allows us to obtain the second-order nonlinear susceptibility and thus the vibrational response function. Without pump pulses at $400 \mathrm{~nm}$, the inverse Fourier transformation of the vibrational response function reveals a free induction decay of vibrational polarization of $\mathrm{C}-\mathrm{O}$ stretching created by a short infrared pulse. The free induction decay is perturbed when an intense 400 -nm pump pulse following the infrared pulse is irradiated, because diffusion and desorption of $\mathrm{CO}$ are induced by the pump pulse. The time evolution of instantaneous $\mathrm{C}-\mathrm{O}$ stretching frequency retrieved from the perturbed free induction decay shows a redshift followed by a rapid reverse shift when the fluence of pump pulse is high enough to desorb CO. This indicates that the frustrated modes of $\mathrm{CO}$ is first substantially excited and then the parallel momentum of $\mathrm{CO}$ is converted to the normal one through mutual collisions, leading to substantial excitation of the external stretching mode of CO. () 2012 American Institute of Physics. [http://dx.doi.org/10.1063/1.4733720]
\end{abstract}

\section{INTRODUCTION}

Infrared-visible sum frequency generation (IV-SFG) has been used as a powerful method for probing vibrational structures and dynamics of molecules at surfaces and interfaces. ${ }^{1,2}$ Because SFG is governed by the second-order nonlinear susceptibility $\chi^{(2)}$, SF signals are generated only at surfaces or interfaces of materials with centrosymmetry, where the symmetry is inherently broken; thus, this makes IV-SFG a surface sensitive vibrational spectroscopy. Because the spectroscopy is performed with ultrashort laser pulses: either narrow band ps infrared (IR) and visible pulses or broadband fs IR and ps visible pulses, this spectroscopy is useful for probing nuclear dynamics at surfaces including vibrational relaxation, site-tosite hopping, and desorption of adsorbates when used as a probe in pump-probe measurements.

Take CO adsorbed on metal surfaces as a typical example. An ultrashort pump pulse excites substrate electrons to create hot electrons. Nonadiabatic couplings between hot electrons and $\mathrm{CO}$ nuclear motions excite the vibrational modes of $\mathrm{CO}$ with respect to the surface, i.e., the frustrated modes. As the fluence of pump pulses increases, the frustrated modes are more strongly excited, resulting in hopping and desorption of $\mathrm{CO}$. The diffusion and desorption probabilities show strong nonlinearity on the pump fluence. This feature can be interpreted in terms of desorption induced by multiple electronic transitions (DIMET). ${ }^{3-10}$ Anharmonic couplings between the internal $\mathrm{C}-\mathrm{O}$ stretching and the

\footnotetext{
a) Author to whom correspondence should be addressed. Electronic mail:
} matsumoto@kuchem.kyoto-u.ac.jp. frustrated modes manifest themselves in transient frequency shifts of the $\mathrm{C}-\mathrm{O}$ stretching mode. ${ }^{11-13}$ Therefore, the $\mathrm{C}-\mathrm{O}$ stretching frequency is a good indicator for probing diffusion and desorption dynamics.

Bonn and co-workers ${ }^{14}$ studied the hopping dynamics of $\mathrm{CO}$ on a $\operatorname{Pt}(533)$ surface under strong excitation of substrate electrons with a 130 -fs pump pulse at $800 \mathrm{~nm}$ by measuring transient changes in the relative intensities of the stretching bands of $\mathrm{CO}$ on step and terrace sites. They demonstrated that the excitation of the frustrated rotation (FR) is more effective than that of the frustrated translation (FT) in promoting siteto-site hopping. The coupling time of the FR mode of $\mathrm{CO}$ on $\operatorname{Pt}(111)$, i.e., the time scale on which energy flows between the substrate electrons and the FR mode, was reported to be $0.1-0.3$ ps $^{13-15}$ This implies that the frustrated rotation can be excited substantially while the surface is irradiated by a 130 -fs pump pulse. Thus, it is necessary to monitor instantaneous $\mathrm{C}-\mathrm{O}$ stretching frequency even in the pulse duration of the pump pulse for a better understanding of site-to-site hopping and desorption dynamics. This requires a substantially high time resolution.

In conventional time-resolved SFG employed in previous studies, a time-evolving vibrational polarization created by a broadband IR pulse is convoluted with a long visible pulse to generate SFG signals. Thus, the transient SFG spectra are determined by the evolution of vibrational polarization within the duration of the visible pulse; this limits the time resolution. Here, we propose another way of monitoring the dynamics of diffusion and desorption with a high time resolution. Recently, we have applied the phase-sensitive detection in time-resolved sum frequency generation spectroscopy 
to probe nonadiabatic coupling of $\mathrm{CO}$ adsorbed on $\mathrm{Pt}(111) .{ }^{16}$ This detection method allows us to obtain both the amplitude and phase of the vibrational response function of the sample $R_{\mathrm{v}}^{(1)}(\omega)$. Once we obtain this, we can transform $R_{\mathrm{v}}^{(1)}(\omega)$ into the time domain $R_{\mathrm{v}}^{(1)}(t)$ with inverse Fourier transformation. Since the temporal profile of $R_{\mathrm{v}}^{(1)}(t)$ contains all the vibrational dynamic information of adsorbate, we can analyze this with a greater time resolution.

This paper describes the measurements of instantaneous frequency shifts of the internal stretching of $\mathrm{CO}$ on $\operatorname{Pt}(111)$ under strong pump pulse irradiation at $400 \mathrm{~nm}$. As a result of strong electronic excitation of the substrate, a substantial amount of $\mathrm{CO}$ adsorbates is excited into precursor states for diffusion and desorption. By using time-resolved SFG with phase-sensitive detection and analyzing perturbed free induction decays, we obtain the spectral features of precursors.

\section{EXPERIMENT}

Experiments were performed with an ultrahigh vacuum (UHV) chamber (base pressure $<2 \times 10^{-10}$ Torr). A Pt (111) single crystal was cleaned by cycles of $\mathrm{Ar}^{+}$sputtering, annealing $(1000 \mathrm{~K})$, and oxygen treatment $\left(1 \times 10^{-7}\right.$ Torr, $800 \mathrm{~K})$.

An optical setup for time-resolved IV-SFG with phasesensitive detection was described in detail previously. ${ }^{16}$ Briefly, the output pulse of a Ti:sapphire regenerative amplifier (Spectra physics, $1 \mathrm{kHz}, 1.8 \mathrm{~mJ} /$ pulse) was split into three to generate a broadband IR pulse (150-fs duration, $7 \mu \mathrm{J} /$ pulse, centered at $\sim 2100 \mathrm{~cm}^{-1}$ ), a narrow band $800 \mathrm{~nm}$ "visible" pulse (1-ps duration, $15 \mu \mathrm{J} /$ pulse), and a 400-nm pump pulse (150-fs duration). The delay of the visible pulse with respect to the IR pulse $t_{\mathrm{IR}}$ was fixed at $600 \mathrm{fs}$. These p-polarized pulses were focused onto the sample surface and refocused onto a local oscillator ( $\mathrm{LO}$, a GaAs wafer) placed outside the UHV chamber with spherical mirrors. A time interval between a SF signal from the sample surface and that from the local oscillator $t_{\mathrm{L}}$ was adjusted to be 3 ps with a quartz plate inserted in the optical path of the signal beam from the sample. Spectral interferograms were recorded by using a polychromator with a charge-coupled device camera.

The average sample temperature was kept at $90 \mathrm{~K}$ during irradiation with laser pulses. The absorbed fluence of 400-nm pump pulses $I_{\mathrm{p}}$ was varied from 30 to $130 \mathrm{~J} / \mathrm{m}^{2}$. At $I_{\mathrm{p}}$ $>40 \mathrm{~J} / \mathrm{m}^{2}$, CO coverage was depleted as a result of photodesorption. We estimated a fraction of coverage desorbed per a pump pulse to be $6 \times 10^{-5} \mathrm{ML}$ at $I_{\mathrm{p}}=90 \mathrm{~J} / \mathrm{m}^{2}$ and $2 \times 10^{-3}$ ML at $I_{\mathrm{p}}=130 \mathrm{~J} / \mathrm{m}^{2}$, assuming the first-order desorption kinetics. Here, $1 \mathrm{ML}$ corresponds to $1.5 \times 10^{19}$ atoms $/ \mathrm{m}^{2}$. To maintain a fractional coverage at $\sim 0.3 \mathrm{ML}$ during the measurements, we continuously exposed the sample to $\mathrm{CO}$ at a constant pressure in the range from $2 \times 10^{-8}$ to $5 \times 10^{-7}$ Torr, depending on the fluence of pump pulses.

\section{METHOD}

Sum frequency (SF) signals are generated through the second-order process upon irradiation of visible $\left(\omega_{1}\right)$ and IR $\left(\omega_{2}\right)$ light, so that the signal intensity $I(\omega)$ is given as,

$$
\begin{aligned}
I(\omega) & \propto\left|E_{3}^{(2)}(\omega)\right|^{2} \\
& \propto\left|\chi^{(2)}\left(\omega ; \omega_{1}, \omega_{2}\right) E_{1}\left(\omega_{1}\right) E_{2}\left(\omega_{2}\right)\right|^{2},
\end{aligned}
$$

where $\omega=\omega_{1}+\omega_{2}, \chi^{(2)}\left(\omega ; \omega_{1}, \omega_{2}\right)$ is the second-order nonlinear susceptibility, $E_{3}^{(2)}(\omega), E_{1}\left(\omega_{1}\right)$, and $E_{2}\left(\omega_{2}\right)$ are the electric field components of SF, visible, and IR pulses, respectively.

We can describe the emitted SF signal field in the time domain in terms of the second-order response function $R^{(2)}\left(t_{2}, t_{1}\right),{ }^{17}$

$$
\begin{aligned}
E_{3}^{(2)}\left(t ; t_{\mathrm{IR}}\right) \propto & \int_{0}^{\infty} d t_{2} \int_{0}^{\infty} d t_{1} R^{(2)}\left(t_{2}, t_{1}\right) E_{1}\left(t-t_{2} ; t_{\mathrm{IR}}\right) \\
& \times E_{2}\left(t-t_{2}-t_{1}\right) .
\end{aligned}
$$

Here, $t_{1}$ is the time elapsed following the IR, and $t_{2}$ is the time elapsed following the visible pulse, and the signal field is parametrically dependent on the delay between the peak maxima of the visible and IR pulses $t_{\mathrm{IR}}$. Because the electronic response of bulk metal with respect to the visible pulse dephases rapidly, it can be assumed to be instantaneous. In addition, if the IR pulse duration is much shorter than the dephasing time of a vibrational mode of interest, the electric field of IR pulse can be approximated as $E_{2}(t)$ $\approx \delta(t)$. Under these assumptions, the SF signal field can be approximated as

$$
\begin{aligned}
E_{3}^{(2)}\left(t ; t_{\mathrm{IR}}\right) & \propto E_{1}\left(t ; t_{\mathrm{IR}}\right) \cdot R_{\mathrm{v}}^{(1)}(t) \\
& =\bar{E}_{1}(t)\left\{\exp \left(i \omega_{1} t\right)+\text { c.c. }\right\} \cdot R_{\mathrm{v}}^{(1)}(t),
\end{aligned}
$$

where $R_{\mathrm{v}}^{(1)}(t)$ is the vibrational response of the sample and $\bar{E}_{1}(t)$ is the slowly varying envelope of the visible pulse. Thus, a complete measurement of $E_{3}^{(2)}(t)$ allows us to obtain the time evolution of $R_{\mathrm{v}}^{(1)}(t)$. In the frequency domain, Fourier transform of Eq. (3) leads to

$$
E_{3}^{(2)}(\omega) \propto \bar{E}_{1}\left(\omega-\omega_{1}\right) \otimes R_{\mathrm{v}}^{(1)}\left(\omega-\omega_{1}\right),
$$

where $\otimes$ is the convolution operator. Thus, $E_{3}^{(2)}(\omega)$ is proportional to $R_{\mathrm{v}}^{(1)}\left(\omega-\omega_{1}\right)$ provided that the spectral width of the visible pulse is sufficiently narrow.

We employed phase-sensitive detection of $E_{3}^{(2)}(t)$ by using Fourier-transform spectral interferometry. ${ }^{18,19}$ The SF signal from the sample $E_{3, \mathrm{~S}}^{(2)}(t)$ is superimposed on that from a local oscillator $E_{3, \mathrm{~L}}^{(2)}(t)$ delayed by $t_{L}$ with respect to $E_{3, \mathrm{~S}}^{(2)}(t)$. The power spectrum of the total field $I(\omega)$ is

$$
\begin{aligned}
I(\omega) \propto & \left|E_{3, \mathrm{~S}}^{(2)}(\omega)+E_{3, \mathrm{~L}}^{(2)}(\omega)\right|^{2} \\
= & \left|E_{3, \mathrm{~S}}^{(2)}(\omega)\right|^{2}+\left|E_{3, \mathrm{~L}}^{(2)}(\omega)\right|^{2} \\
& +2 \operatorname{Re}\left[E_{3, \mathrm{~S}}^{(2)}(\omega) E_{3, \mathrm{~L}}^{(2) *}(\omega) \exp \left(i \omega t_{\mathrm{L}}\right)\right] .
\end{aligned}
$$

Here, $E_{3, \mathrm{~S}}^{(2)}(\omega)$ and $E_{3, \mathrm{~L}}^{(2)}(\omega)$ are the Fourier components of $E_{3, \mathrm{~S}}^{(2)}(t)$ and $E_{3, \mathrm{~L}}^{(2)}(t)$, respectively. While $\left|E_{3, \mathrm{~S}}^{(2)}(\omega)\right|^{2}$ and $\left|E_{3, \mathrm{~L}}^{(2)}(\omega)\right|^{2}$ give SF signals from the sample and LO, respectively, the last term $2 \operatorname{Re}\left[A_{\mathrm{SL}}(\omega)\right]$ $=2 \operatorname{Re}\left[E_{3, \mathrm{~S}}^{(2)}(\omega) E_{3, \mathrm{~L}}^{(2) *}(\omega) \exp \left(i \omega t_{\mathrm{L}}\right)\right]$ results in interference between the two. As in Figure 1(a), a typical SF spectrum 

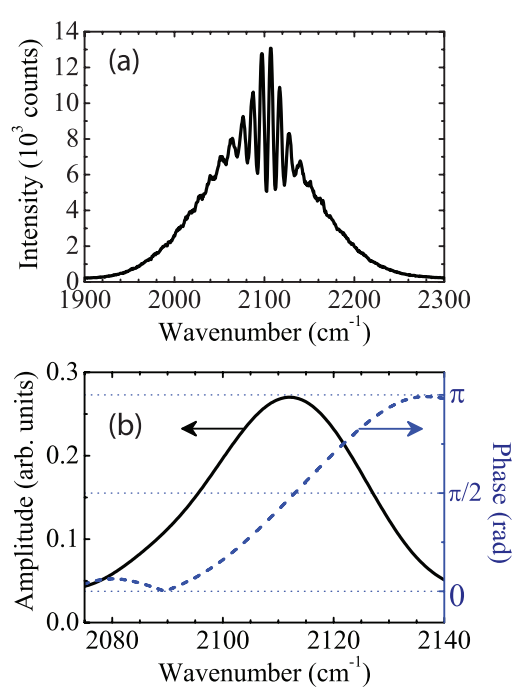

FIG. 1. (a) Typical SF spectrum obtained from $\mathrm{Pt}(111)$ covered with $\mathrm{CO}$, showing clearly the interference superimposed on the SF signals from the sample and local oscillator. (b) The amplitude (solid black) and phase (dashed blue) of the vibrational response function $R_{\mathrm{v}}^{(1)}\left(\omega-\omega_{1}\right)$ obtained from the spectrum in (a).

around $2100 \mathrm{~cm}^{-1}$ obtained from the $\operatorname{Pt}(111)$ surface covered with CO clearly shows the interference superimposed on the individual SF contributions of the sample and LO.

The complex field $A_{\mathrm{SL}}(\omega)$ can be obtained by the following procedure:

$$
A_{\mathrm{SL}}(\omega) \propto \hat{F}\left[\theta\left(t-t^{\prime}\right) \hat{F}^{-1}[I(\omega)]\right],
$$

where $\hat{F}$ and $\hat{F}^{-1}$ imply Fourier and inverse Fourier transform, respectively, and $\theta\left(t-t^{\prime}\right)$ is the Heaviside function. The value of $t^{\prime}$ is set to be in between 0 and $t_{L}$ so as to retain a time domain feature originating in $A_{S L}(\omega)$ and to eliminate an unwanted feature at around $t=0$ originating in $\left|E_{3, \mathrm{~S}}^{(2)}(\omega)\right|^{2}$ and $\left|E_{3, \mathrm{~L}}^{(2)}(\omega)\right|^{2}$.

Measurements with a reference sample, a GaAs wafer located at the position of the Pt crystal in the UHV chamber, provided $A_{\mathrm{RL}}(\omega)=E_{3, \mathrm{R}}^{(2)}(\omega) E_{3, \mathrm{~L}}^{(2) *}(\omega) \exp \left(i \omega t_{\mathrm{L}}\right)$, where $E_{3, \mathrm{R}}^{(2)}(\omega)$ is a complex electric field of SF from the GaAs wafer. The susceptibility of the reference $\chi_{\mathrm{R}}^{(2)}=\left|\chi_{\mathrm{R}}^{(2)}\right| \exp \left[i \phi_{\mathrm{R}}\right]$ is almost constant in the IR frequency region measured. Thus, a ratio spectrum $r(\omega)=A_{\mathrm{SL}}(\omega) / A_{\mathrm{RL}}(\omega)=E_{3, \mathrm{~S}}^{(2)}(\omega) / E_{3, \mathrm{R}}^{(2)}(\omega)$ is proportional to $\left|R_{\mathrm{v}}^{(1)}\left(\omega-\omega_{1}\right)\right| \exp \left[i\left(\phi_{\mathrm{S}}-\phi_{\mathrm{R}}\right)\right]$. Because $\phi_{\mathrm{R}}$ does not depend on frequency, we chose the unknown parameter $\phi_{\mathrm{S}}$ in such a way that the phase becomes zero at the lower frequency side of $\mathrm{CO}$ stretching band under no pump irradiation as illustrated in Figure 1(b). Using $R_{\mathrm{v}}^{(1)}\left(\omega-\omega_{1}\right)$ extracted with this procedure, we can obtain $R_{\mathrm{v}}^{(1)}(t)$ with inverse Fourier transformation.

\section{RESULTS AND DISCUSSION}

Measurements were performed at various delay times $t_{\mathrm{d}}$ of a 400-nm pump pulse with respect to the IR pulse, while a time interval between the IR and visible pulses for SFG was fixed at $600 \mathrm{fs}$. At $t_{\mathrm{d}}=-10 \mathrm{ps}$, i.e., the pump pulse is delayed by $10 \mathrm{ps}$ from the IR pulse, the vibrational polarization

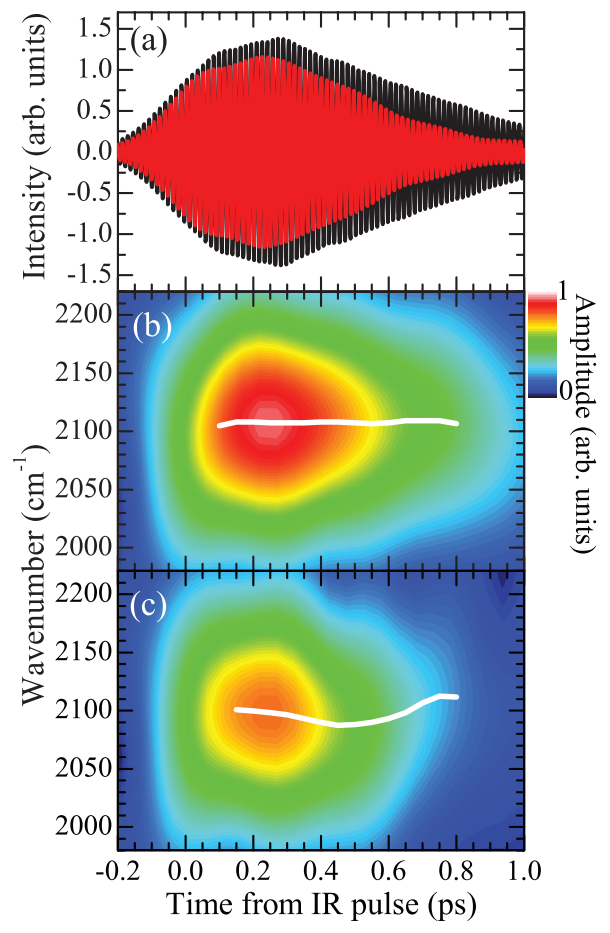

FIG. 2. (a) The time profile of IR-pulse-induced vibrational polarization retrieved from the inverse Fourier transformation of $R_{\mathrm{v}}^{(1)}\left(\omega-\omega_{1}\right)$ at the time delay of a $400-\mathrm{nm}$ pump pulse with respect to an IR pulse: $t_{\mathrm{d}}=-10 \mathrm{ps}$ (black) and $-0.3 \mathrm{ps}$ (red). Spectrograms of the vibrational polarization are obtained by using the time window of a Gaussian profile with a half width of 150 fs: (b) $t_{\mathrm{d}}=-10 \mathrm{ps}$ and (c) $-0.3 \mathrm{ps}$. The peak frequencies are indicated with white lines. The fluence of the pump pulse was $130 \mathrm{~J} / \mathrm{m}^{2}$.

induced by the IR pulse is not perturbed by the pump pulse. Thus, we recovered a free induction decay of vibrational polarization by inverse Fourier transformation of $R_{\mathrm{v}}^{(1)}\left(\omega-\omega_{1}\right)$ as shown in Figure 2(a) (black line). We converted the vibrational polarization into the frequency-time space using the time window of a Gaussian profile with a half width of 150 fs: time-dependent spectral amplitudes (spectrograms). As expected, the instantaneous peak frequency of $\mathrm{CO}$ stretching in the spectrogram at $t_{\mathrm{d}}=-10 \mathrm{ps}$ has no influence of pump irradiation as shown in Figure 2(b).

When $t_{\mathrm{d}}$ is close to $0 \mathrm{ps}$, the vibrational polarization is significantly modulated by the pump pulse. A perturbed free induction decay and its spectrogram at $t_{\mathrm{d}}=-0.3 \mathrm{ps}$ are depicted in Figure 2(a) (red line) and 2(c), respectively. The peak frequency is modulated particularly at around $t_{\mathrm{d}}$ $=-0.3 \mathrm{ps}$ and the vibrational polarization dephases faster than that at $t_{\mathrm{d}}=-10 \mathrm{ps}$.

Figure 3 shows the pump fluence dependence of the peak frequency of $\mathrm{C}-\mathrm{O}$ stretching. The delay of a pump pulse was fixed at $t_{\mathrm{d}}=-0.3 \mathrm{ps}$. Below $I_{\mathrm{p}}=40 \mathrm{~J} / \mathrm{m}^{2}$, the peak frequency shifts downward; the amount of redshift increases with the pump fluence. This observation is consistent with previous studies. $^{12,13}$ As the pump fluence increases, the peak frequency shifts more steeply to red at around $0.3 \mathrm{ps}$. At above $I_{\mathrm{p}}$ $=80 \mathrm{~J} / \mathrm{m}^{2}$ where photo-stimulated desorption of $\mathrm{CO}$ occurs effectively, a new feature appears: it shows a turning point at around $150 \mathrm{fs}$ after pump pulse irradiation, followed by rapid increase in its frequency. This phenomenon was not observed 


\section{Time from pump pulse (ps)}

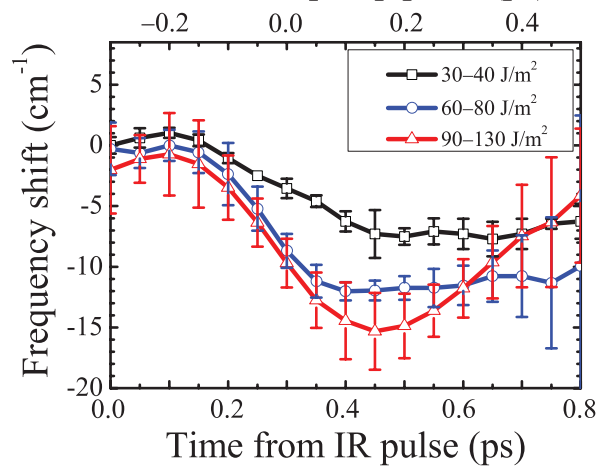

FIG. 3. Pump fluence dependence of the frequency at the peak of $\mathrm{C}-\mathrm{O}$ stretching band. The top horizontal axis denotes the delay time after the 400-nm pump pulse, while the bottom axis denotes the delay time after the IR pulse that creates vibrational coherence. In these measurements, the pump pulse was irradiated at $0.3 \mathrm{ps}$ after the IR pulse. Plotted are the frequency shifts averaged over several data sets obtained within the range of the fluence of a 400-nm pump pulse cited in the figure.

in the previous conventional time-resolved SFG studies performed with much lower pump fluences.

We simulated the stretching frequency shifts in terms of anharmonic couplings between $\mathrm{C}-\mathrm{O}$ stretching and $\mathrm{CO}-$ frustrated modes. Assuming that the populations of frustrated modes fluctuate due to energy exchange with substrate through electron-phonon coupling, we can express the $\mathrm{C}-\mathrm{O}$ stretching frequency as $^{20,21}$

$$
\omega(t)=\omega_{0}+\sum_{i} \alpha_{i}\left\langle n_{i}(t)\right\rangle
$$

Here, $\omega_{0}$ is the fundamental frequency of $\mathrm{C}-\mathrm{O}$ stretching at $T=0 \mathrm{~K}, \alpha_{i}$ is the anharmonic coupling constant between the $i$ th frustrated mode and the $\mathrm{C}-\mathrm{O}$ stretching mode, and $\left\langle n_{i}(t)\right\rangle$ is the statistical average of the population of the $i$ th frustrated mode. We further assumed that: (1) the electron temperature $T_{e}(t)$ is described by the two-temperature model ${ }^{22}$ with the parameters cited in Ref. 11; (2) the energy distribution of $i$ th vibrational mode is statistical with a time-varying adsorbate temperature $T_{a, i}(t)$, while the vibrational mode interacts with substrate electrons through a coupling constant $\eta_{a, i}$,

$$
\frac{d T_{a, i}(t)}{d t}=\eta_{a, i}\left\{T_{e}(t)-T_{a, i}(t)\right\} .
$$

Figure 4 shows the results simulated with the parameters tabulated in Table I. In the low fluence range: $I_{\mathrm{p}} \leq 40 \mathrm{~J} / \mathrm{m}^{2}$, the monotonous redshifts of $\mathrm{C}-\mathrm{O}$ stretching frequency were well simulated under the assumption of linear shifts due to the anharmonic couplings with FT and FR modes represented by Eq. (7) (dashed black line in Figure 4). In contrast, it was impossible to simulate the peak frequency shift in the range $I_{\mathrm{p}}$ $>60 \mathrm{~J} / \mathrm{m}^{2}$ by Eq. (7): because the values of $\alpha_{i}$ of FT and FR modes are negative, the frequency shifts steadily with delay time (dotted blue line in Figure 4).

So far we have not included the anharmonic coupling with the Pt-CO external stretching (ES) mode. The coupling constant for this mode should be positive, because the internal stretching frequency of $\mathrm{CO}$ on $\operatorname{Pt}(111), 2104 \mathrm{~cm}^{-1}$, approaches that of $\mathrm{CO}$ in the gas phase, $2143 \mathrm{~cm}^{-1}$, as the

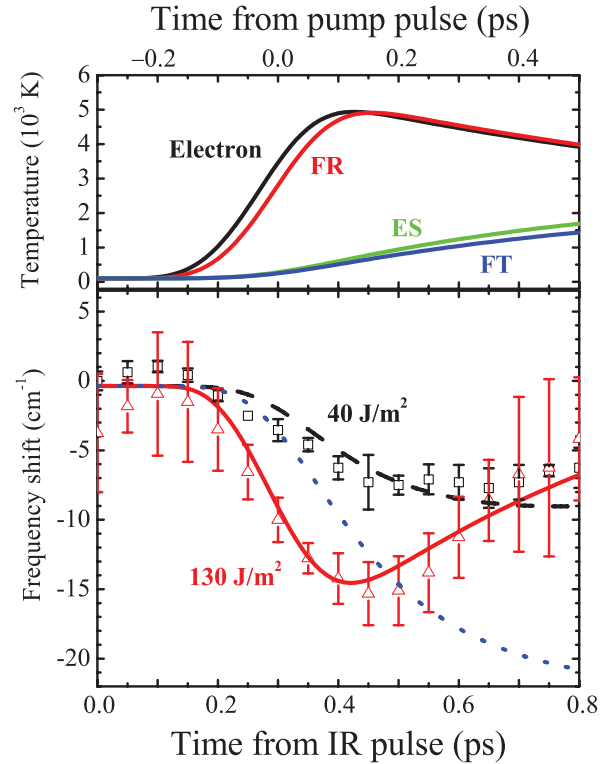

FIG. 4. Simulated results of the frequency shifts of $\mathrm{C}-\mathrm{O}$ stretching at the pump fluence of 40 and $130 \mathrm{~J} / \mathrm{m}^{2}$ in comparison with the observed ones. The 400 -nm pump pulse was irradiated at 0.3 ps after the IR pulse that creates vibrational coherence. The lower panel shows experimental results and simulated results. While the redshifts observed at $40 \mathrm{~J} / \mathrm{m}^{2}$ (open squares) were well simulated through anharmonic couplings with FR and FT (black dashed line), the simulation at $130 \mathrm{~J} / \mathrm{m}^{2}$ with the same coupling constants (blue dotted line) deviates significantly from the observed results (open triangles). The observed results at $130 \mathrm{~J} / \mathrm{m}^{2}$ are well simulated by including anharmonic coupling with the external CO stretching mode (red solid line). The parameters used in the simulations are in Table I. The upper panel shows the time profiles of temperatures of electron, frustrated rotation, frustrated translation, and external CO stretching, calculated with the parameters used in the simulation of the results at $130 \mathrm{~J} / \mathrm{m}^{2}$.

Pt-CO external stretching mode is excited. Taking into account this anharmonic coupling together with couplings with FT and FR, we could simulate the reversed shift starting at $0.4 \mathrm{ps}$ after the IR pulse at the high fluence, $I_{\mathrm{p}}=130 \mathrm{~J} / \mathrm{m}^{2}$ (solid red line in Figure 4) reasonably well with the parameters given in Table I.

Note that the strong enhancement in the coupling with the FR mode is required to simulate the steep redshift; the friction coefficient increases by an order of magnitude with increase of the pump fluence from $I_{\mathrm{p}}=40$ to $130 \mathrm{~J} / \mathrm{m}^{2}$. This can be interpreted as the change in the coupling scheme between substrate electrons and adsorbate nuclear motions. At the low fluence, hot electrons near the Fermi level play a major role in the nonadiabatic coupling: the excitation of

TABLE I. Parameters used in the simulations of peak frequencies of $\mathrm{C}-\mathrm{O}$ stretching as a function of delay time.

\begin{tabular}{lccc}
\hline \hline Mode $^{\mathrm{a}}$ & $\begin{array}{c}\text { Fluence } \\
\left(\mathrm{J} / \mathrm{m}^{2}\right)\end{array}$ & $\begin{array}{c}\alpha \\
\left(\mathrm{cm}^{-1}\right)\end{array}$ & $\begin{array}{c}1 / \eta_{a, i} \\
(\mathrm{ps})\end{array}$ \\
\hline FR & 40 & -2.0 & 0.2 \\
FT & & -0.5 & 1.5 \\
FR & & -2.0 & 0.02 \\
FT & 130 & -0.5 & 1.5 \\
ES & & +7.0 & 1.2 \\
\hline \hline
\end{tabular}

${ }^{\mathrm{a} F R}$ : frustrated rotation; FT: frustrated translation; ES: external stretching. 
nuclear motion can be accounted for with the electron friction model. In contrast, at the high fluence, hot electrons bring the adsorbate onto a highly electronically excited potential energy surface. The center-of-mass motions of adsorbate are initiated at the excited state before the adsorbate is quenched back to the ground potential energy surface. Many cycles of excitation and deexcitation cause effective excitation of frustrated modes of adsorbate. The nonadiabatic coupling in this regime is described with DIMET. Brandbyge et al. ${ }^{23}$ developed the theory including the transition from the friction to DIMET regime, demonstrating that the coupling coefficient increases with pump fluence particularly in the DIMET regime.

In the anharmonic coupling scheme, the very effective nonadiabatic coupling between FR and substrate electrons is necessary to take into account the steep and rapid redshift of the $\mathrm{C}-\mathrm{O}$ stretching mode, as mentioned above. Another way to explain the rapid redshift is more direct: the weakening the $\mathrm{C}-\mathrm{O}$ bond by filling of the unoccupied bands of $\mathrm{CO}$. The unoccupied $5 \sigma_{a}$ and $2 \pi_{a}^{*}$ were reported to be at 2.51 and $4.3 \mathrm{eV}$ above the Fermi level, respectively. ${ }^{24-26}$ The high electron temperatures created by strong pump pulses allow hot electrons to resonantly fill the unoccupied states; thus, the population gain particularly in the $2 \pi_{a}^{*}$ state changes the $\mathrm{C}-\mathrm{O}$ bond strength and the stretching frequency. Arnolds and coworkers proposed the electron transfer from the substrate to adsorbate contributes to the redshifts of stretching modes of $\mathrm{NO}$ and $\mathrm{CO}$ on $\operatorname{Ir}(111)$ under irradiation of pump pulses at $800 \mathrm{~nm} \cdot{ }^{27,28}$ If this electron transfer effect is a dominating contribution to the observed redshifts of $\mathrm{C}-\mathrm{O}$ stretching, the frequency shift will mimic the time evolution of electron temperature. Although the amount of redshift until $0.4 \mathrm{ps}$ after the IR pulse correlates well with $T_{e}$, the discrepancy between the two is evident after 0.4 ps: the amount of redshift decreases faster than $T_{e}$. This discrepancy arises from the contribution of excitation of external $\mathrm{CO}$ stretching, which increases the $\mathrm{C}-\mathrm{O}$ stretching frequency via anharmonic coupling.

Our results indicate that the external $\mathrm{CO}$ stretching is effectively excited as the FR mode is highly excited. While the potential barrier for desorption of $\mathrm{CO}$ on $\mathrm{Pt}(111)$ along the surface normal is $1.5 \mathrm{eV}$, the energy barrier for hopping to the adjacent adsorption sites along the surface plane is in the range from 0.2 to $0.3 \mathrm{eV}$, depending on $\mathrm{CO}$ coverage. ${ }^{29}$ Because $\mathrm{CO}$ is desorbed effectively at $I_{\mathrm{p}}>40 \mathrm{~J} / \mathrm{m}^{2}, \mathrm{CO}$ adsorbates are substantially excited to the states over this barrier. It has been proposed that the FR mode is effective to stimulate site-to-site hopping. ${ }^{14}$ This result was interpreted theoretically as CO hopping is induced by indirect heating of the FT mode by the FR mode. ${ }^{30}$ Therefore, the lateral motion of $\mathrm{CO}$ is substantially excited in the high fluence range. Because the coverage of $\mathrm{CO}$ is close to the saturation, the $\mathrm{CO}$ adsorbates excited over the barrier collide with each other to convert lateral momentum to normal momentum; thus, the collisions enhance the excitation of the external $\mathrm{CO}$ stretching mode, resulting in the reverse shift after 0.4 ps. Molecular dynamics simulations of laser-induced desorption of $\mathrm{CO}$ on $\mathrm{Cu}(100)$ clearly demonstrate this effective momentum conversion by collisions between the energetic adsorbate and its neighbors at $0.5 \mathrm{ML}$ CO coverage. ${ }^{31}$

\section{CONCLUSION}

We probed vibrational dynamics of $\mathrm{CO}$ on $\mathrm{Pt}(111)$ induced by $400-n m$ pump pulses. The resonant spectrum of $\chi^{(2)}(\omega)$ measured by using IV-SFG with phase-sensitive detection is used to obtain the time profiles of vibrational response created by an IR pulse. We investigated dynamics of precursor states for diffusion and desorption. When the fluence of pump pulse was low, the frequency of $\mathrm{C}-\mathrm{O}$ stretching showed a monotonic redshift after the pump pulse. In contrast, when the fluence is substantially high to desorb $\mathrm{CO}$, the frequency of $\mathrm{C}-\mathrm{O}$ stretching shows a much faster redshift followed by a reverse shift. The significant increase in the coupling coefficient with FR responsible for the faster redshift is likely the manifestation of a transition of nonadiabatic coupling scheme: from electron friction to DIMET. The effective coupling with FR results in substantial excitation of adsorbates, leading to collisions of $\mathrm{CO}$ adsorbates; the lateral momentum of precursor states for desorption is converted to the normal momentum. Thus, the anharmonic coupling with the external $\mathrm{CO}$ stretching modes causes the rapid reverse shift observed after around 150 fs after the pump pulse.

\section{ACKNOWLEDGMENTS}

This work was supported by the JST-PRESTO program and Grants-in-Aid for Scientific Research (S) (Grant No. 17105001) and JSPS Fellows (Grant No. 22 -990) from the Japanese Society for the Promotion of Sciences. K.I. is grateful for the JSPS fellowship.

${ }^{1}$ X. D. Zhu, H. Suhr, and Y. R. Shen, Phys. Rev. B 35, 3047 (1987).

${ }^{2}$ Y. R. Shen, The Principles of Nonlinear Optics (Wiley, New Jersey, 2003).

${ }^{3}$ J. A. Prybyla, T. F. Heinz, J. A. Misewich, M. T. Loy, and J. H. Glownia, Phys. Rev. Lett. 64, 1537 (1990).

${ }^{4}$ J. A. Prybyla, H. W. K. Tom, and G. D. Aumiller, Phys. Rev. Lett. 68, 503 (1992).

${ }^{5}$ L. M. Struck, L. J. Richter, S. A. Buntin, R. R. Cavanagh, and J. C. Stephenson, Phys. Rev. Lett. 77, 4576 (1996).

${ }^{6}$ M. Bonn, S. Funk, C. Hess, D. N. Denzler, C. Stempfl, M. Scheffler, M. Wolf, and G. Ertl, Science 285, 1042 (1999).

${ }^{7}$ D. N. Denzler, C. Frischkorn, C. Hess, M. Wolf, and G. Ertl, Phys. Rev. Lett. 91, 226102 (2003).

${ }^{8}$ L. Bartels, F. Wang, D. Möller, E. Knoesel, and T. F. Heinz, Science 305, 648 (2004).

${ }^{9}$ K. Stépán, J. Güdde, and U. Höfer, Phys. Rev. Lett. 94, 236103 (2005).

${ }^{10}$ M. Lawrenz, K. Stépán, J. Güdde, and U. Höfer, Phys. Rev. B 80, 075429 (2009).

${ }^{11}$ T. A. Germer, J. C. Stephenson, E. J. Heilweil, and R. R. Cavanagh, J. Chem. Phys. 98, 9986 (1993).

${ }^{12}$ F. Fournier, W. Zheng, S. Carrez, H. Dubost, and B. Bourguignon, Phys. Rev. Lett. 92, 216102 (2004).

${ }^{13}$ F. Fournier, W. Zheng, S. Carrez, H. Dubost, and B. Bourguignon, J. Chem. Phys. 121, 4839 (2004).

${ }^{14}$ E. H. G. Backus, A. Eichler, A. W. Kleyn, and M. Bonn, Science 310, 1790 (2005).

${ }^{15}$ E. H. G. Backus, M. Forsblom, M. Persson, and M. Bonn, J. Phys. Chem. C 111, 6149 (2007).

${ }^{16}$ K. Watanabe, K. Inoue, I. F. Nakai, and Y. Matsumoto, Phys. Rev. B 81, 241408 (2010).

${ }^{17}$ J. E. Laaser, W. Xiong, and M. T. Zanni, J. Phys. Chem. B 115, 2536 (2011).

${ }^{18}$ L. Lepetit, G. Chériaux, and M. Joffre, J. Opt. Soc. Am. B 12, 2467 (1995).

${ }^{19}$ S. Nihonyanagi, S. Yamaguchi, and T. Tahara, J. Chem. Phys. 130, 204704 (2009).

${ }^{20}$ B. N. J. Persson and R. Ryberg, Phys. Rev. B 40, 10273 (1989). 
${ }^{21}$ T. A. Germer, J. C. Stephenson, E. J. Heilweil, and R. R. Cavanagh, J. Chem. Phys. 101, 1704 (1994).

${ }^{22}$ S. I. Anisimov, B. L. Kapeliovich, and T. L. Perel'man, Sov. Phys. JETP 39, 375 (1974).

${ }^{23}$ M. Brandbyge, P. Hedegård, T. F. Heinz, J. A. Misewich, and D. M. Newns, Phys. Rev. B 52, 6042 (1995).

${ }^{24}$ K. C. Chou, S. Westerberg, Y. R. Shen, P. N. Ross, and G. A. Somorjai, Phys. Rev. B 69, 153413 (2004).

${ }^{25}$ V. Dose, J. R. M. Bradshaw, and K. C. Prince, Surf. Sci. 179, 90 (1987).
${ }^{26}$ T. Anazawa, I. Kinoshita, and Y. Matsumoto, J. Electron Spectrosc. Relat. Phenom. 88-91, 585 (1998).

${ }^{27}$ I. M. Lane, Z.-P. Liu, D. A. King, and H. Arnolds, J. Phys. Chem. C 111, 14198 (2007).

${ }^{28}$ H. Arnolds, Prog. Surf. Sci. 86, 1 (2011).

${ }^{29}$ E. Schweizer, B. N. J. Persson, M. Tüshaus, D. Hoge, and A. M. Bradshaw, Surf. Sci. 213, 49 (1989).

${ }^{30}$ M. Hayashi, Y. Ootsuka, M. Paulsson, B. N. J. Persson, and H. Ueba, Phys. Rev. B 80, 245409 (2009).

${ }^{31}$ C. Springer and M. Head-Gordon, Chem. Phys. 205, 73 (1996). 